\title{
Deonomastika von Städte- und Ländernamen aus lexikographischer Perspektive
}

\author{
Björn Rothstein (Stuttgart)
}

\begin{abstract}
The paper argues for a special lexicographic treatment of the derivates of city and country names. "Normally", proper names are not part of a dictionary, they are only considered in the encyclopedia. Therefore, their derivates should not figure in the dictionary from a semantic point of view. In Swedish and French, the derivates of those proper names turn however out to be morphologically irregular. They should therefore be part of the dictionary. The paper compares their lexicographic treatment in Swedish and French dictionaries.
\end{abstract}

\section{$1 \quad$ Einleitung}

Die vorliegende Arbeit untersucht aus kontrastiver Perspektive die lexikographische Behandlung von Bewohnernamen zu Städten, Ländern und Kontinenten. Miteinander verglichen werden das Schwedische und das Französische. Untersucht wird, in welchen Typ von Nachschlagewerken diese Deonomastika aufzunehmen sind und wie ihre Integration im Einzelnen erfolgt. In einem ersten Schritt werden die verschiedenen Typen von Nachschlagewerken skizziert, dann sollen Eigenschaften von Eigennamen erarbeitet und anschließend ihre Derivate semantisch und morphologisch im Hinblick auf ihre lexikographische Behandlung untersucht werden. Ein empirischer Teil beschließt die Arbeit.

\section{Definitionen}

Häufig werden Nachschlagewerke in Lexika und Wörterbücher unterteilt. Nachschlagewerke, die über die Sprache informieren, werden Wörterbücher, solche, die über die Welt, das Außersprachliche, informieren, Lexikon genannt. Wörterbücher konzentrieren sich damit auf das Wort und seine Beziehung zu anderen Wörtern, Lexika auf Individuen oder Objekte (vgl. Hausmann (1977: 13), Lara (1989: 280), Hupka (1989: 990), Engelberg/Lemnitzer (2001: 11f) und Jackson (2002: 21)). Diese intuitiv zunächst einleuchtende Unterteilung von Nachschlagewerken fällt in der Praxis jedoch oftmals unscharf aus, da die exakte Trennung zwischen Sprache und Welt schwierig, wenn nicht unmöglich ist. Beispielsweise kann eine Vielzahl von Wörtern sowohl dem Wörterbuch als auch dem Lexikon zugeordnet werden. Aspirin kann etwa als ein Warenprodukt und damit als ein Objekt oder als ein „Wort“ mit der Bedeutung Kopfwehtablette aufgefasst werden. Im ersten Fall sollte es im Lexikon, im zweiten Fall im Wörterbuch stehen. Solche Unterscheidungsschwierigkeiten haben teilweise dazu geführt, die Trennung zwischen Lexikon und Wörterbuch abzulehnen. Gleichwohl ergibt sich das Problem, wie zwischen Sprach- und Sachlichkeit überhaupt sinnvoll unterschieden werden kann: um die Bedeutung des Worts Löwe angeben zu können, muss man wissen, was ein Löwe ist. Und damit wird es nötig, harte Trennlinien zwischen außersprachlichem und sprachlichem Wissen zu ziehen. Haimann (1980: 331) behauptet etwa, dass eine solche Unterscheidung nicht möglich sei und dass Wörterbücher auch Lexika seien. Dennoch haben 
viele Lexikographen die Unterscheidung beibehalten und in der Tat zeigen sich deutliche Unterschiede in der Gestaltung von Lexika bzw. Wörterbüchern, auch wenn im Einzelfall die Integration mancher Wörter problematisch sein mag. $\mathrm{Zu}$ den wichtigsten Unterscheidungsmerkmalen zählen:

Lexika erklären erstens die außersprachliche Referenz, Wörterbücher gehen sprachlichen Fragestellungen nach. Das Lexikon teilt demnach mit, was ein Löwe ist, das Wörterbuch, was das Wort Löwe bedeutet (s. Rey-Debove (1971b: 35)). Lexika können zweitens Bilder, Graphiken, Zeichnungen oder Diagramme als Bestandteil ihrer Lemmaartikel haben, in Wörterbüchern sind diese nicht anzutreffen. Drittens geben Wörterbücher im Unterschied zu Lexika soziolinguistische Informationen wie Soziolekt, Stil, Dialekt etc an. Viertens werden nur in Wörterbüchern phonetisch-phonologische, morphologische, syntaktische und semantische/pragmatische Informationen zum betreffenden Lemma gegeben. ${ }^{1}$

Drei Gestaltungsmöglichkeiten ergeben sich aus dieser Perspektive für die Konzeption von Nachschlagewerken: ausschließliche Bearbeitungen von Sach- oder Sprachlichkeit und die Integration beider Aspekte. Die Kombination von Sprach- und Sachlichkeit entspricht der Typologie des Allbuchs. Sprachlichkeit erfordert die Bereitstellung einer grammatischen Metasprache, um die jeweilige grammatische Distribution der zu beschreibenden Lemmata aufführen $\mathrm{zu}$ können. Angaben dieser Art sind v. a. Aussprachedarstellungen, Wortartbestimmung, Bedeutung und Flexionseigenschaften etc. Zur Beschreibung der Sachlichkeit sind diese Angaben nicht nötig, da hier nicht sprachliche, sondern außersprachliche Informationen wie kulturelle, geographische, soziale, historische etc. gegeben werden.

Zwar mag die Unterteilung von Nachschlagewerken in Lexika und Wörterbücher umstritten sein, seltene Einigkeit herrscht jedoch bei der lexikographischen Behandlung von Eigennamen (dazu etwa Haiman (1980), Bergenholtz (1989)). Sie tauchen nicht im Wörterbuch auf, sondern werden im Lexikon aufgeführt. Da sich diese Arbeit nur mit den Ableitungen von Eigennamen, genauer Ortsnamen, beschäftigt, ist es daher legitim, von den zwei Nachschlagewerktypen Lexikon und Wörterbuch im Weiteren auszugehen, auch wenn die Unterscheidung im Allgemeinen schwierig ist. Das Allbuch ist uninteressant für das Ziel dieser Arbeit, da es die Menge der Charakteristika von Lexikon und Wörterbuch trägt. Eigennamen können dort aufgeführt werden, egal, ob sie Teil der Sach- oder Sprachlichkeit sind. Wie Derivate zu Eigennamen sich in Nachschlagewerke einfügen lassen, soll nun genauer untersucht werden. Dabei ist ein Blick auf die semantischen Eigenschaften von Eigennamen notwendig.

Eigennamen referieren, ohne dass der Kontext Spezifizierungen und Erklärungen bereitzustellen hat. Ihr Status ist somit autodeterminativ und noetisch (Leys (1979), Molino (1982) und Knobloch (1992)). Ihre Unfähigkeit zu Synonymie und Antonymie ist ein deutlicher Hinweis hierfür. Die semantische Standardauffassung zu Eigennamen ist etwa bei Kamp/Reyle (1993) nachlesbar (s. auch Kripke (1972), Dowty et al (1981: 192-193), Gamut (1991: 161ff) und Partee (1990: 353)).

Proper names do not refer to their bearers by virtue of describing them, but because of a certain non-descriptive connection which holds between a name and its bearer, a connection reminiscent of that which holds between a label and the object to which it is attached. Kamp/Reyle (1993: 247)

1 Bekanntlich lassen sich Wörterbücher und Lexika in eine Vielzahl von Untertypen untergliedern. Im Folgenden soll eine solche Unterteilung nicht versucht werden, da sie nicht nötig ist. Für die hier gewonnenen Ergebnisse spielt es im Wesentlichen keine Rolle, ob das betreffende Wörterbuch ein- oder mehrsprachig ist. Entscheidend ist nur, dass es sich um ein Wörterbuch handelt. 
Allerdings unterliegen Eigennamen ähnlich den Gattungsnamen gewissen Aktualitätsanforderungen an das gemeinsame Weltwissen zweier Sprecher. Beide müssen das durch einen Eigennamen bezeichnete Objekt kennen. Dies ist jedoch weniger ein Charakteristikum der Eigennamen, sondern vielmehr eine Frage des common grounds, des gemeinsamen Hintergrundswissens zwischen Sprecher und Hörer.

Da Eigennamen auf Außersprachliches referieren, ohne es zu charakterisieren, sind sie in der Regel nicht Bestandteil des Wörterbuchs. Autodeterminativer und noetischer Status verlangen aus semantischer Perspektive keine Wörterbucherklärungen, da nicht semantische Fragegestellungen bei ihrer Definition geklärt werden müssen, sondern die Sachlichkeit betreffende Informationen zu geben sind. Wer sich über Karl XII oder Schweden informieren möchte, wird demnach nicht zum Wörterbuch greifen, sondern im Lexikon in Erfahrung bringen, wer oder was die durch Karl XII bzw. Schweden bezeichneten Dinge oder Individuen sind (vgl. etwa Haimann (1980: 330)). Auch morphosyntaktisch weisen Eigennamen keine Besonderheiten auf, die ihre Aufnahme in das Wörterbuch rechtfertigen würden. Zwar sind ihre Artikel- und Pluralfähigkeiten eingeschränkt (etwa *das Tübingen, aber: das Tübingen der zwanziger Jahre oder *die Tübingens), doch gelten diese Beschränkungen systematisch für die gesamte Klasse der Ortsnamen. Anders verhält es sich, wie noch zu zeigen ist, mit ihren Derivaten.

\section{Derivate zu Eigennamen (Deonomastika)}

Wenn man davon ausgeht, dass Wörterbuchartikel in der Regel zumindest Auskunft über die phonetisch-phonologischen, graphischen, morphosyntaktischen und semantischen Eigenschaften eines Lexems geben, so muss man sich der Frage stellen, ob im Falle der Derivate von Eigennamen (Deonomastika) mindestens eine dieser Eigenschaften zwangsweise ihre Eingliederung in das Wörterbuch verlangt. Weisen die Derivate keinerlei sprachliche Besonderheiten auf und sind ihre Form und Bedeutung durch allgemeine Regeln vorhersagbar, so sind sie lediglich regelhafte, vorhersagbare Ableitungen von Eigennamen und als solche nicht im Wörterbuch aufzuführen. Im Folgenden soll es um morphologische und semantische Aspekte von Deonomastika gehen. Gezeigt wird, dass lediglich morphologische Unregelmäßigkeiten, Idiosynkrasien, die Integration der betreffenden Deonomastika in das Wörterbuch rechtfertigen.

Betrachtet man Deonomastika zu Ortsnamen aus semantischer Sicht, so kann man feststellen, dass sie drei mögliche Bedeutungen aufweisen. Parisien kann "aus Paris kommend" , "zu Paris gehörend" oder "nach Pariser Art" bedeuten. Allgemeiner kann man sagen, dass zu einem Ort "x" die drei Deonomastikabedeutungen "aus x kommend", "zu x gehörend", "nach $\mathrm{x}$-er Art" möglich sind. Entscheidend ist nun, dass keine der drei Bedeutungen die zugrunde liegende Basis semantisch in nennenswerter Weise modifiziert. Die Basis von Deonomastika bleibt stets ein Eigenname. Die drei Bedeutungen sind paraphrasierbar als "kommt aus x", "gehört zu x" und "gemäß der üblichen Art in x". Damit besteht zwischen dem " $\mathrm{x}$ " in den Deonomastika und dem durch die Eigennamen bezeichneten außersprachlichen Referenten "x" kein nennenswerter semantischer Unterschied. Deonomastika sind lediglich um Lokalbzw. Modalausdrücke ergänzte Eigennamen. Aufgrund ihres semantischen Status als Eigennamen gehören Deonomastika daher nicht in das Wörterbuch. Zu diesem Schluss kommt auch Rey-Debove (1971a: 372):

En écartant les noms propres [du dictionnaire de langues, B. R.], on démotive tous leurs dérivés à l'intérieur du dictionnaire. Les adjectifs dérivés de noms propres sont sacrifiés du point de vue sémantique. Rey-Debove (1971a: 372) 
Deonomastika sind aus lexikographischer Perspektive v. a. morphologisch interessant. Kann die Form der Deonomastika durch Kenntnis allgemeiner morphologischer Regeln vorhergesagt werden, d.h. vom entsprechenden Eigennamen abgeleitet werden, ohne dass Zweifel irgendeiner Art bei der Art der Ableitung auftreten, so besteht kein Grund zur Aufnahme in das Wörterbuch. Im Folgenden wird für das Französische und Schwedische gezeigt, dass die morphologische Form von Deonomastika nicht durch allgemeine Regeln vorhersagbar ist.

Im Französischen können Deonomastika zu Ortsnamen durch eine Vielzahl von Suffixen gebildet werden. Wolf (1964) etwa unterscheidet für in Frankreich liegende Städtenamen gebildete Adjektive die Suffixe -ar, -asque, -enc, -ench, -en, -ate, -asse, -iste, -ite, -ote, -ais, ois, -is, -ès, -ain, -ien (+Varianten), -an/-eran, -and, -ant, -ent, -ano, -in, -on (+Varianten), ou, -u, -et, -ot, -at, -as, -eau, -ol, -oy, -ère, -iere/-(uy)er, -al, -ard und -auf sowie einige weitere Suffixe. Schweickard (1992) nennt u. a. bei Deonomastika außerhalb von Frankreich liegenden Ortschaften die Suffixe -ein, -ois, -ain, -an, -in, -on, -ique, -iaque, -ite, -iste, -ate, iate, -(i)ote, -esque, -asque, -ègue (-ène), -teque, -ol, -i, -aque, -and und -er. Dieser großen Menge von Suffixen steht nun eine sehr kleine Zahl unterschiedlicher Funktionen gegenüber, so dass "identische Funktionen durch eine größere Zahl verschiedener Suffixe erfüllt" werden (Schweickard (1992: 67)). Dies wirft, da die unterschiedlichen Suffixe das gleiche ausdrücken, nämlich "zu x-gehörend", "aus x kommend" oder "nach x-er Art", die Frage auf, welcher Ortsnamen welches Suffix selegiert und ob dies Regeln unterliegt. Dazu führt Schweickard (1992: 134) aus:

Gerade im Bereich der Ableitung von Eigennamen ist bei der Frage von Möglichkeit, Unmöglichkeit und Regelhaftigkeit einzelner Bildungsformen prinzipiell Zurückhaltung angebracht.

Stammallomorphie zwischen Eigennamen und Ableitung (vgl. (3)), zahlreiche Doppelformen (s. (1)), Differenzierungen wie in 0 und Entlehnungen aus dem Latein und anderen Sprachen belegen eindrucksvoll die Schwierigkeiten bei der Bildung von Deonomastika zu Städtenamen im Französischen. Hinzu kommen die große Anzahl der oben aufgeführten Suffixe und sprecherindividualistische Varianten. Es besteht daher Grund zur Annahme, dass die Ermittlung der korrekten Form von Deonomastika zur Benutzung eines Nachschlagewerks zwingt (s. a. Schweickard (1992)).

(1)

\author{
Saint-Etienne \\ Saint-Etienne-de-Mont-Luc \\ Saint-Etienne-de-Saint-Geoirs \\ Saint-Etienne-de-Lugdarès \\ Saint-Etienne-de-Tinée
}

Stéphanois

Stéphanois

Stéphanois

Stéphanois

Stéphanois

Barisiens

Barrois

Baralbins

Barois

Bar-Séquanais 


$\begin{array}{ll}\text { Bar-le-Duc } & \text { Barisiens } \\ \text { Besançon } & \text { Bisontin } \\ \text { Poitier } & \text { Poitevin } \\ \text { Fontainebleau } & \text { Bellifontain }\end{array}$

Festzuhalten ist somit, dass die Bildung von Deonomastika zu Städtenamen im Französischen zu einem großen Teil unregelmäßig ist, worauf auch Schweickard (1992: 134) vorsichtig hinweist.

Auch im Schwedischen liegen im Falle der Bildung der Deonomastika von Länder- und Kontinentnamen zahlreiche nicht durch Regeln vorhersagbare Idiosynkrasien vor. Eine detaillierte Untersuchung dazu findet sich bei Sigurd (1965), der darum bemüht ist, Regeln für die Bildung von Deonomastika zu finden, gleichzeitig aber zugeben muss, dass letztlich die Form des nominalen Derivats zu Länder- und Kontinentnamen niemals hundertprozentig vorhersagbar ist (S. 232). Sigurd untersucht 200 Nationalitätsbezeichnungen samt Ableitungen und unterteilt sie in 14 verschiedene morphologische Muster, die unter anderem durch Analogie bedingt sind. Zwar lassen sich in der Tat verschiedene Bildungstypen unterscheiden, doch bleiben zahlreiche Unregelmäßigkeiten bestehen. Beispielsweise heißt der Bewohner von Tyskland (Deutschland) tysk, der von England engelsman und der von Finland finländare. Weiter gibt es asiat zu Asien, belgier zu Belgien und italienare zu Italien sowie brasilian zu Brasilien. Außerdem liegt ein reiches Allomorphieangebot vor. Unter anderem -are, -er, -an, -at, -iak, -man und -ing sind mögliche Derivationssuffixe mit der Bedeutung "Bewohner von $x "$. Die sprachliche Unsicherheit bei der Bildung von Deonomastika zeigt sich auch in den zahlreichen Doppelformen wie isländare und islänning als Bewohner von Island. Weitere Formen und Unregelmäßigkeiten können der Tabelle am Ende des Artikels entnommen werden.

Die hier untersuchte Gruppe von Deonomastika weist demnach sowohl im Schwedischen wie im Französischen morphologische Idiosynkrasien auf. Damit besteht ein Grund zur Aufnahme in das Wörterbuch. Während die Semantik der Deonomastika regelhaft ist und Deonomastika semantisch daher nur erweiterte Eigennamen sind (sofern sie die obigen Bedeutungen aufweisen), ist ihre Form nicht vorhersagbar und muss daher nachgeschlagen werden. Zwar ist nicht ausgeschlossen, dass sie im Lexikon aufgeführt werden können, doch ist das eigentliche Anliegen, mit dem man sich an das Nachschlagewerk wendet, nicht die Frage "was ist", sondern "wie bildet man". Da die Form hier relevant ist und nicht die Definition, erzwingt ihre morphologische Unregelmäßigkeit die Aufnahme ins Wörterbuch. Zu einem ähnlichen Ergebnis kommt übrigens auch Becker (1990: 188) für das Deutsche.

\section{Aufnahmeverfahren in Nachschlagewerke}

Die Aufnahmepraxis von Deonomastika in französische Wörterbücher ist v. a. von Schweickard (1989/1992) ausführlich beschrieben worden. Daher wird im Folgenden nur ein knapper Überblick ohne aufwendige empirische Ausrichtung gegeben. Im Falle der schwedischen Wörterbücher gilt jedoch, dass die hier untersuchte Fragestellung bisher unbeantwortet geblieben ist. Dementsprechend wird empirisch vorgegangen.

Für das Französische lassen sich generell drei Arten von Aufnahmeverfahren von Deonomastika feststellen. Die erste Gruppe, zu denen Wörterbücher wie der Petit Robert (PR) 
und der Grand Robert (GR) zählen, nimmt Derivate zu Städtenamen in separaten Listen am Wörterbuchende auf, sie integriert jedoch teilweise die Derivate in die alphabetische Makrostruktur des Wörterbuchs. Eine zweite Gruppe verzeichnet lediglich die (relativen) Derivate zu Städtenamen in einer der Makrostruktur angehängten Liste am Wörterbuchende wie es z.B. der Dictionnaire du Français Contemporain (DFC) tut. Die dritte Gruppe umfaßt solche Wörterbücher, die völlig auf Listen verzichten. Hierher gehören der Grand Larousse de la langue française (GLLF) und der Trésor de la langue française (TLF).

Der hier mitüberprüfte Petit Larousse (PLil) ist nicht als Wörterbuch, sondern als Allbuch zu verstehen. Auf ihn wird am Ende des Abschnitts eingegangen.

\begin{tabular}{|l|c|c|c|c|c|c|c|c|c|}
\hline & \multicolumn{2}{l}{} & \multicolumn{2}{l}{ GR } & TLF & GLLF & \multicolumn{2}{l|}{ PLil } & \multicolumn{2}{l|}{ DFC } \\
\hline & Lemma & Liste & Lemma & Liste & Lemma & Lemma & Lemma & Lemma & Liste \\
\hline Avignonnais & - & + & - & + & + & - & - & - & + \\
\hline Dijonnais & - & + & - & + & - & + & - & - & + \\
\hline Havrais & - & + & - & + & - & - & - & - & + \\
\hline Lyonnais & - & + & + & + & + & + & - & - & + \\
\hline Marseillais & + & + & + & + & + & + & + & + & + \\
\hline Nancéien & - & + & - & + & + & - & - & - & + \\
\hline Niçois & - & + & + & + & + & + & + & - & + \\
\hline Poitevin & + & + & + & + & + & + & + & - & + \\
\hline Strasbourgeois & - & + & - & + & + & - & - & - & + \\
\hline Toulousain & - & + & + & + & + & + & - & - & + \\
\hline
\end{tabular}

Tab. 1

Im Annex zum GR finden sich Listen zu Derivaten von Personennamen, unter der Berücksichtigung, ob diese gelebt haben oder nur fiktiv sind. Eine weitere Liste mit Ortsnamen ist ebenfalls aufgeführt. Der GR bietet für beide Listen alphabetisch zwei Zugriffsmöglichkeiten an. Sie sind entweder nach den Eigennamen selbst oder nach ihren Derivaten geordnet, was die Suche nach "Auf"- oder Ableitung ermöglicht. In Fällen wie Poitevin bieten sich solche Listen an, da weder von der Ableitung auf die Basis noch von der Basis auf die Ableitung geschlossen werden kann. Im Falle des GR sind alle zehn hier untersuchten französischen Deonomastika in die Liste aufgenommen. Fünf von ihnen tauchen jedoch auch in der alphabetischen Makrostruktur des Wörterbuchs auf: Marseillais, Lyonnais, Niçois, Poitevin und Toulousain. Für die ersten beiden werden neben der Bewohnerbedeutung noch weitere Bedeutungen angegeben: La Marseillaise (Nationalhymne) und Sauce lyonnaise. Bei beiden Ausdrücken handelt es sich um Eigennamen. Es ist jedoch nicht einsichtig, warum Derivate zu Lyon, Marseille, Nice, Poitiers und Toulouse in der Makrostruktur verzeichnet sind, Ableitungen zu Strasbourg oder Nancy fehlen.

Was für den GR gesagt wurde, lässt sich auch auf den PR übertragen. Wiederum ist nicht einsichtig, warum Derivate zu Poitiers und Marseille in der Makrostruktur verzeichnet sind. Die alphabetische Liste des PR ist proportional kleiner als die des GR. Für beide 
Wörterbücher gilt, dass die Aufnahme von Derivaten zu Ortsnamen unsystematisch motiviert ist.

Der DFC aus der zweiten Gruppe verzeichnet alle hier untersuchten Derivate in einer separaten alphabetischen Liste am Wörterbuchende. In die Makrostruktur wurde nur Marseillais aufgenommen, wohl um den Eigennamen La Marseillaise einzuführen.

Auch der TLF verfährt unsystematisch. Unverständlich bleibt, warum Bezeichnungen wie Havrais ausgespart, solche wie Poitevin aufgenommen wurden. Havrais ist wie Poitevin eine unregelmäßige Bildung, da der Artikel von Le Havre getilgt wird bei der Derivation. Eine morphologische Argumentation für die Aufnahme von Poitevin ist somit nicht stichhaltig.

Ähnliches gilt für den GLLF. Auch hier ist die Aufnahme unsystematisch. Avignon, Le Havre und Strasbourg wurden weggelassen, alle anderen aufgenommen. Wie die Auswertung von Tab. 1 tendenziell zeigt, ist Schweickards (1989/1992) Analyse unbedingt zuzustimmen. Die Aufnahmepraxis von Derivaten zu Eigennamen ist durch und durch unsystematisch betrieben. Es spiegelt sich die Problematik der Theorie in der Praxis wieder.

Der PLil verfährt nicht weniger unsystematisch. Da es sich beim PLil jedoch um ein Allbuch mit starker Selektion handelt, dürfte dies damit erklärbar sein. Zu fragen bliebe, wie ein Allbuch mit extensiver Makrostruktur die Problematik der Aufnahme behandeln würde.

Zusammenfassend lässt sich sagen, dass die hier beschriebenen Aufnahmeverfahren in den französischen Wörterbüchern häufig inkonsequent und unsystematisch sind. Eine vernünftige Aufnahmepraxis müßte erstens Kriterien für die Aufnahme oder die Ablehnung der Deonomastika in die Makrostruktur angeben, zweitens diese mit völliger Konsequenz befolgen und drittens deutliche Unterschiede zwischen der Bedeutungsgruppe "aus $\mathrm{x}$ kommend", "zu x gehörend" sowie "nach x-er Art" und darüber hinausgehende Bedeutungen wie La Marseillaise machen. Als Lösung bieten manche französische Wörterbücher separate Listen am Ende der Makrostruktur an, die lediglich die Formen ohne eigene Mikrostruktur auflisten.

$\mathrm{Zu}$ den untersuchten schwedischen Nachschlagewerken zählen: Bonniers svenska ordbok (3. bis 8. Auflage), Stora ordboken, Norstedts svensk ordbok (3.), Norstedts Svensk ordbok och svensk uppslagsbok (1. und 2.), Norstedts Plusordbok, Nusvensk ordbok, Ordbok öfver svenska språket, Svenska akademiens ordlista (1. bis 12.) und Svensk synonym ordbok. Mit Nordstedts Plusordbok und Norstedts Svensk ordbok och svensk uppslagsbok (1. und 2.) liegen Allbücher vor. Bei den anderen Nachschlagewerken handelt es sich um Wörterbücher im obigen Sinn. Untersucht wurden die Derivate zu den in der Tabelle am Ende dieses Artikels aufgeführten Länder- und Kontinentnamen. Einige der Derivate haben neben der Bewohnerbedeutung noch eine zusätzliche, etwa Amerikanare als großes Auto. Diese Formen werden zwar aufgeführt, aber nicht weiter unter dem hier interessierenden lexikographischen Aspekt beachtet. Die Auswahl der Ländernamen ist in gewisser Weise jedoch willkürlich, obwohl darauf geachtet wurde, für Schweden kulturell, wirtschaftlich, politisch oder geographisch wichtige Länder zu untersuchen.

Im Wesentlichen lassen sich drei Integrationsstrategien der Länder- und Kontinentnamen in den schwedischen Nachschlagewerken ausmachen. Erstens lassen einige Wörterbücher die Länder- und Kontinentnamen und ihre Derivate vollkommen aus (etwa Svensk Synonym Ordbok und Svensk ordbok). Zweitens werden die besagten Derivate ohne den Eigennamen integriert wie in SAOL (1. bis 9. Auflage), Bonniers (3. bis 8.), Nordstedts (3.) und Stora ordboken. Drittens integrieren die Allbücher Plus und Svensk ordbok och uppslagsbok (1. und 2.) die Derivate und die dazugehörigen Eigennamen. Die Aufnahme von Eigennamen 
begründet sich durch die Konzeption dieser Nachschlagewerke als Allbücher. Eine vierte Strategie findet sich in SAOL (10. bis 12.). Dort werden nur die irregulär gebildeten Derivate zu Städtenamen aufgeführt. Gewöhnlich werden im Schwedischen durch Suffigierung mit -bo an den Städtenamen Bewohnernamen gebildet. Da die Bildung von Bewohnernamen zu Ländernamen stets einer gewissen Irregularität unterliegt (dazu Sigurd (1965: 232)), ist eine solche Regelung für diese Derivate nicht möglich.

Auch die schwedischen Nachschlagewerke erweisen sich in der Behandlung der besagten Derivate im Detail als unsystematisch. Die Selektion der aufgeführten Bewohnernamen der Länder ist nicht nachvollziehbar. Sie scheint weder wirtschaftlich, geographisch, politisch noch kulturell begründbar. Betrachtet man beispielsweise Stora Ordboken, so finden sich zahlreiche Ungereimtheiten bei der Integration von Bewohnernamen zu Ländern und Kontinenten. Es kamen Afrikand, Amerikanare, Australier und Europé, nicht jedoch Asiat vor. Bei Ländern fehlen Engelsman, Isländare, Italienare, Svensk, Tsyk und Österrikare, aber nicht Brasilian und Fransos. Der Leser kann sich leicht von weiteren Unsystematizitäten anhand der Tabelle am Ende dieses Artikels überzeugen.

Der Vergleich der Behandlung von Deonomastika zu Städte-, bzw. Länder- und Kontinentnamen in den schwedischen und französischen Nachschlagewerken ist nun in zweierlei Hinsicht aufschlussreich. Während schwedische Lexikographen im Falle der Städtenamen nur die irregulären Formen in die Makrostruktur des Wörterbuchs integrieren, verfolgen ihre französischen Kollegen eine andere Strategie: Deonomastika werden in manchen Wörterbüchern tabellarisch ohne Mikrostruktur am Ende des Nachschlagewerks aufgelistet. Von der schwedischen Lexikographie ist ein solcher Vorschlag meines Wissens nie unterbreitet worden. Problematisch an der schwedischen Strategie ist nun, dass ein etwaiges Fehlen des betreffenden Derivats in der Makrostruktur fälschlicherweise dahingehend interpretiert wird, dass diese Form regulär ist. Hier scheint die französische Strategie gelungener.

\section{$5 \quad$ Zusammenfassung}

Viele französische Wörterbücher greifen als Lösung auf das Anhängen separater Listen mit Deonomastika ohne erklärende Mikrostruktur zurück. Die alphabetische Makrostruktur des Wörterbuchs wird durch Listen mit Derivaten am Wörterbuchende ergänzt. Dabei liegen keinerlei Erklärungen vor, eingegangen wird nur auf die morphologische Relation zwischen Ortsname und Ableitung. Diese Vorgehensweise stimmt also völlig überein mit der Hauptthese dieser Arbeit: da die Semantik die Derivate zu Ortsnamen eindeutig dem Lexikon, die morphologische Komponente jedoch dem Wörterbuch zuweist, muss ein Weg gefunden werden, der beidem gerecht wird. Die Aufnahme der Deonomastika mit regelhafter Bedeutung "aus x kommend", "zu x gehörend" und "nach x-er Art" in die Makrostruktur eines Wörterbuchs ist aus semantischer Perspektive überflüssig. Da die Bedeutung durch Kenntnis allgemeiner Regeln vorhersagbar ist, muss sie nicht im Wörterbuch aufgeführt werden. Die Aufnahme der Derivate in die Makrostruktur würde zudem die Kapazität eines Wörterbuchs sprengen. Aus ökonomischen und praktischen Gründen wie Platzsparen werden also separate Listen bereitgestellt, deren Anlegung ja durchaus die Morphologie des Deonomastikums klärt. Ein entsprechendes Verfahren ist in den schwedischen Nachschlagewerken bisher nicht unterbreitet worden, obwohl die morphologischen Voraussetzungen im Falle von Deonomastikabildungen zu Ländernamen den französischen Bildungen zu Städtenamen sehr ähnlich sind. 


\section{Erklärungen zur Tabelle am Ende des Artikels:}

$\begin{array}{ll}\text { (m.) } & \text { maskulin } \\ \text { (f.) } & \text { feminin } \\ - & \text { betreffendes Wort erscheint nicht in Nachschlagewerk } \\ + & \text { betreffendes Wort erscheint in Nachschlagewerk } \\ \text { Sv. O. / U. } & \text { Svensk Ordbok/Uppslagsbok } \\ \text { PLUS } & \text { Norstedts Plusordbok }\end{array}$

Appelative Verwendungen:

Amerikanare (stor bil) großes amerikanisches Auto

Finne (liten vårblåsa) Aknepickel

Fransyska (vårstycke) Art von Braten

Kanadensare (öpen kanot) Kanu

\section{Literaturangaben}

Becker, Thomas (1990): Analogie und morphologische Theorie. München.

Bergenholtz, Henning (1989): "Probleme der Selektion im allgemeinen einsprachigen Wörterbuch". In: Hausmann, Franz Josef (1989) et al (eds.): 773-779.

Dowty, David R./Wall, Robert E./Stanley, Peters (1981): Introduction to Montague semantics. Dordrecht.

Engelberg, Stefan/Lemnitzer, Lothar (2001): Lexikographie und Wörterbuchbenutzung. Tübingen.

Gamut, L.T.F. (1991): Intensional logic and logical grammar. Logic, language and meaning, vol. 2. Chicago.

Haimann, John (1980): "Dictionaries and encyclopedias". Lingua 50: 329-357.

Hausmann, Franz Josef (1977): Einführung in die Benutzung der neufranzösischen Wörterbücher. Tübingen.

Hausmann, Franz Josef/Reichmann, Oskar/Wiegand, Herbert Ernst/Zgusta, Ladislav (eds.) (1989): Wörterbücher: ein internationales Handbuch zur Lexikographie Band 1. Berlin.

Hupka, Werner (1989): "Das enzyklopädische Wörterbuch". In: Hausmann, Franz Josef et al (eds.) (1989): 988-999.

Jackson, Howard (2002): Lexicography. An introduction. London.

Kamp, Hans / Reyle, Uwe (1993): From discourse to logic. Dordrecht.

Knobloch, Clemens (1992): "Eigennamen als Unterklasse der Nomina und in der Technik des Sprechens". Sprachwissenschaft 17: 451-473.

Kripke, Saul (1972): "Naming and necessity". In: Davidson, Donald/Harman, Gilbert (eds.): Semantics of natural language. Dordrecht: 253-355.

Lara, Luis Fernando (1989): "Dictionnaire de langue, encyclopédie et dictionnaire encyclopédique: le sens de leur distinction". In: Hausmann, Franz Josef et al (1989): 280287.

Leys, Odo (1979): "Was ist ein Eigenname? Ein pragmatisch orientierter Standpunkt". Leuvense bijdragen 68, 61-68.

Molino, Jean (1982): "Le nom propre dans la langue". Language 66: 5-20. 
Partee, Barbara H./Meulen, Alice G.B. ter/Wall, Robert E. (1990), Mathematical methods in linguistics. Dordrecht.

Rey-Debove, Josette (1971a): "Limites des applications de la linguistique à la lexicographie (Dictionnaires de langues monolingues)". In: Perren, George E./Trim, John (eds.) Applications of Linguistics. Selected papers of the Second International Congress of Applied Linguistics Cambridge 1969. Cambrigde: 369-375.

Rey-Debove, Josette (1971b): Étude linguistique et sémiotique des dictionnaires français contemporains. The Hague.

Schweickard, Wolfgang (1989): "Le traitement des formations déonomastiques dans la lexicographie française". In: Kremer, Dieter (1988- ) (ed.): Actes du XVIII Congrès International de Linguistique et de Philologie Romanes (Université de Trier). Tübingen: 242-253.

Schweickard, Wolfgang (1992): "Deonomastik". Ableitungen auf der Basis von Eigennamen im Französischen (unter vergleichender Berücksichtigung des Italienischen, Rumänischen und Spanischen). Tübingen.

Sigurd, Bengt (1965): "Morfologiska mönster i nationalitetsord". Arkiv för nordisk filologi 80: 231-276.

Wolf, Heinz Jürgen (1964): Die Bildung der französischen Ethnica (Bewohnernamen). Genève.

\section{Nachschlagewerke}

\section{Französisch:}

Dubois, Jean et al. (eds.) (1971): Dictionnaire du français contemporain. Paris.

Garnier, Yves et al (eds.) (2000): Le Petit Larousse Illustré. Paris.

Guilbert, Louis (1971): Le Grand Larousse de la langue française en 6 volumes. Paris.

Imbs, Paul (ab 1971): Trésor de la langue française. Paris.

Rey, Alain (ed.) (1985): Le grand Robert de la langue française. Dictionnaire alphabétique et analogique de la langue française. $2^{\mathrm{e}}$ éd. entièrement revue et enrichie. Paris.

Rey-Debove, Josette et al. (eds.) (2000): Le Nouveau Petit Robert. Paris.

\section{Schwedisch:}

Allén, Sture et al (1999): Norstedts svensk ordbok. 3. Auflage. Stockholm.

Axelson, Per/Josephson, Håkan (eds.) (1997): Norstedts Plusordbok. Göteborg universitet: Språkdata. Stockholm.

Collinder, Björn (1983): Stora ordboken. Svensk ordnyckel. Stockholm.

Dalin, Anders Fredrik (1850-55): Ordbok öfver svenska språket. Stockholm.

Göteborgs universitet: Språkdata (1997): Norstedts Svenska ordbok och svensk uppslagsbok. Stockholm. (Sv. O. / U).

Göteborgs universitet: Språkdata (1997/1999): Norstedts Svenska ordbok och svensk uppslagsbok. 2. Auflage. Stockholm. (Sv. O. / U).

Malmström, Sten et al (1980/1986): Bonniers svenska ordbok. 3. Auflage. Stockholm.

Malmström, Sten et al (1980/1991): Bonniers svenska ordbok. 5. Auflage. Stockholm.

Malmström, Sten et al (1980/1994): Bonniers svenska ordbok. 6. Aufl. Stockholm.

Malmström, Sten et al (1980/1998): Bonniers svenska ordbok. 7. Auflage. Stockholm.

Malmström, Sten et al (1980/2002): Bonniers svenska ordbok. 8. Auflage. Stockholm.

Östergren, Olof/Zetterholm, Margareta/Holm, Gösta (1919-1972): Nusvensk ordbok. Stockholm. 
Svenska Akademien (ed.) (1874): Svenska akademiens Ordlista öfver Svenska språket. 1. Auflage. Stockholm. (= SAOL 1).

Svenska Akademien (ed.) (1874): Svenska akademiens Ordlista öfver Svenska språket. 2. Auflage. Stockholm. (= SAOL 2).

Svenska Akademien (ed.) (1875): Svenska akademiens Ordlista öfver Svenska språket. 3. Auflage. Stockholm. (= SAOL 3).

Svenska Akademien (ed.) (1880): Svenska akademiens Ordlista öfver Svenska språket. 4. Auflage. Stockholm. (= SAOL 4).

Svenska Akademien (ed.) (1883): Svenska akademiens Ordlista öfver Svenska språket. 5. Auflage. Stockholm. (= SAOL 5).

Svenska Akademien (ed.) (1889): Svenska akademiens Ordlista öfver Svenska språket. 6. Auflage. Stockholm. (= SAOL 6).

Svenska Akademien (ed.) (1900): Svenska akademiens Ordlista öfver Svenska språket. 7. Auflage. Stockholm. (= SAOL 7).

Svenska Akademien (ed.) (1927): Svenska akademiens ordlista över svenska språket. 8. Auflage. Stockholm. (= SAOL 8).

Svenska Akademien (ed.) (1959): Svenska akademiens ordlista över svenska språket. 9. Auflage. Stockholm. (= SAOL 9).

Svenska Akademien (ed.) (1973): Svenska akademiens ordlista över svenska språket. 10. Auflage. Stockholm. (= SAOL 10).

Svenska Akademien (ed.) (1986): Svenska akademiens ordlista över svenska språket. 11. Auflage. Stockholm. (= SAOL 11).

Svenska Akademien (ed.) (1998): Svenska akademiens ordlista över svenska språket. 12. Auflage. Stockholm. (= SAOL 12).

Swedenborg, Lillemor et al (1992): Svensk synonym ordbok. Stockholm. 


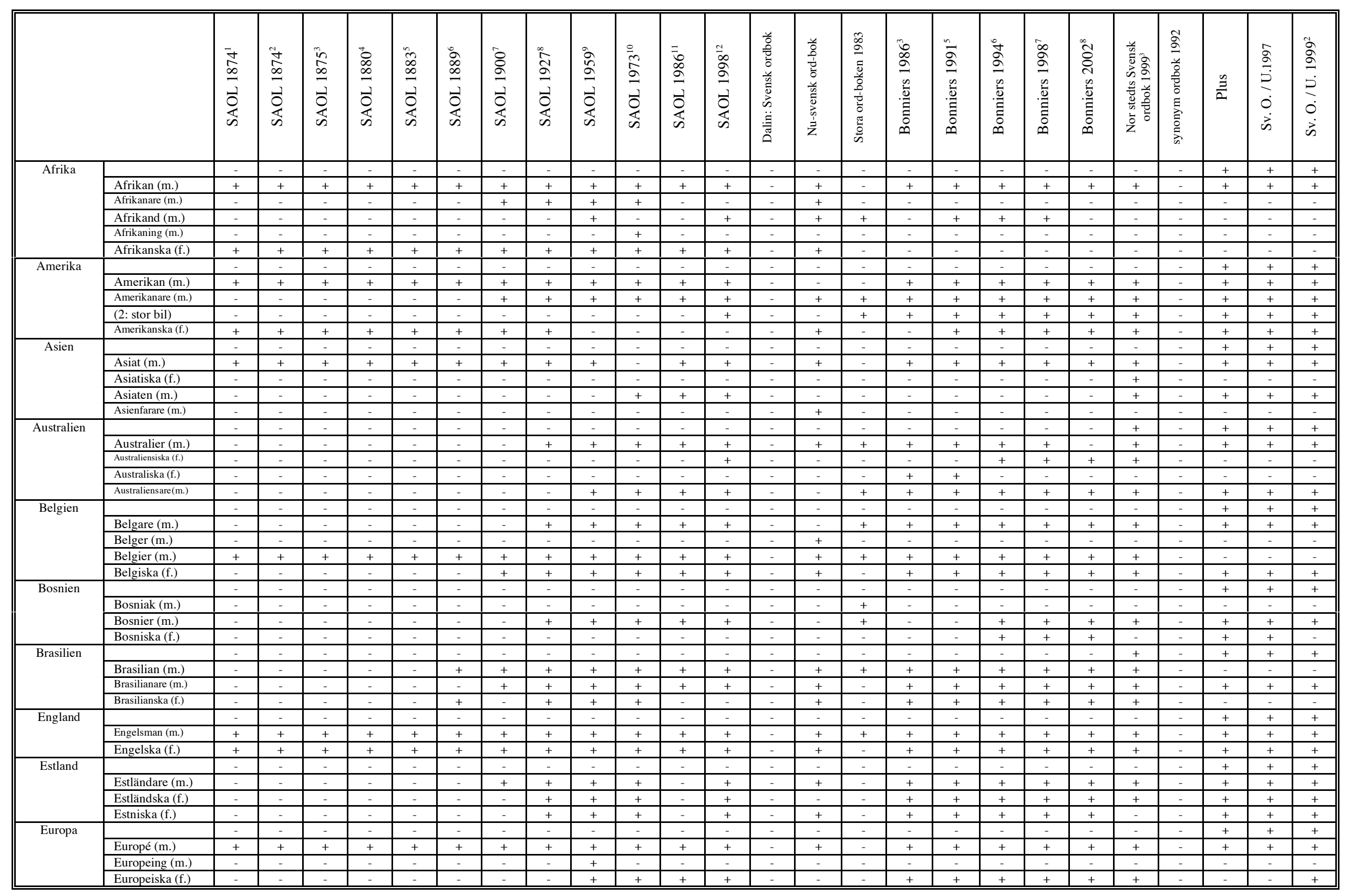



aus lexikografischer Perspektive.

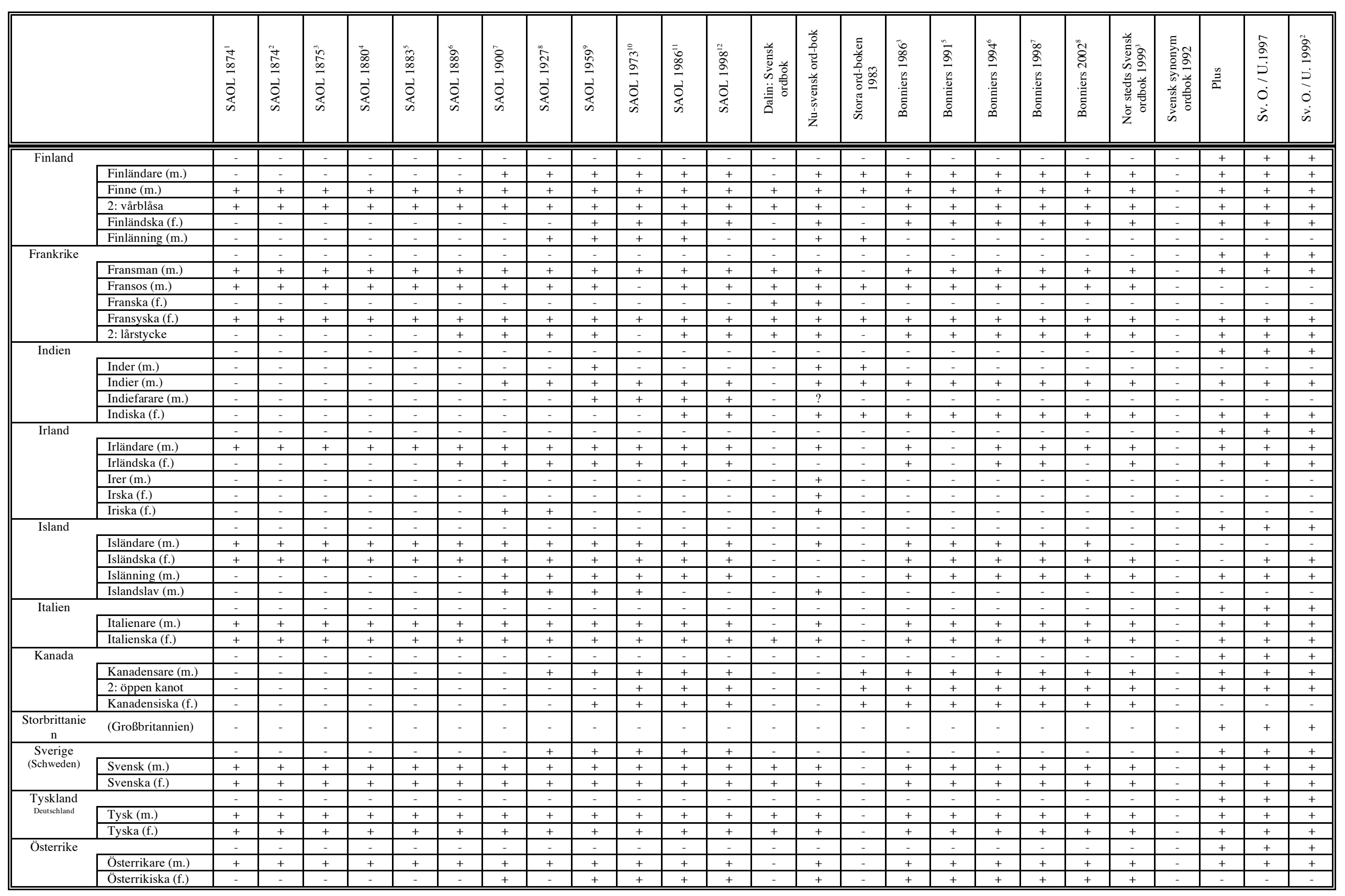

